\title{
IgE: a regulator of autoimmune inflammation in lupus
}

IgE is an important regulator of inflammation and disease pathology in systemic lupus erythematosus (SLE), according to a new study. "Our most significant finding is that $\operatorname{IgE}$ is involved in amplifying the immune response by enhancing the recruitment of immune cells into secondary lymphoid organs," explains researcher Juan Rivera. "The absence of IgE delays the onset of inflammation and the development of disease including kidney pathology associated with lupus nephritis. In humans, the presence of autoreactive $\operatorname{IgE}$ increases the probability of having active disease and lupus nephritis."

Earlier work by Rivera and colleagues had shown that IgE and basophils contribute to the disease phenotype. To study the role of IgE in disease development and autoimmune inflammation, the researchers crossed IgE-deficient mice with either $F c \gamma I I B^{-/-}$ mice, which develop spontaneous lupus, or with $F_{c} \gamma I I B^{-/-}$. Yaa mice, which develop a particularly aggressive form of lupus.
"We chose these models because FcyIIB polymorphisms have been associated with human lupus, and because Yaa functions via TLR7, an innate immune receptor implicated in the development of lupus," explains Rivera.

\section{4 ...the presence of} autoreactive lgE increases the probability of having active disease and lupus nephritis $\%$

Deletion of IgE increased survival, decreased renal deposition of $\mathrm{C} 3$ complement and IgG immune complex, and decreased glomerulonephritis. IgE deletion also normalized plasma cell numbers and the ratio of $\mathrm{CD}^{+}: \mathrm{CD}^{+} \mathrm{T}$ cells in the spleen and lymph nodes. Numbers of dendritic cells, monocytes, neutrophils and activated basophils were increased in the spleen and lymph nodes of mice with lupus, but were normalized to wild-type levels by IgE deficiency. In patients with SLE, the researchers identified an association between the presence of autoreactive IgE and active disease or lupus nephritis.

The researchers acknowledge that a number of questions remain unanswered. "One question of immediate importance is whether IgE solely amplifies the inflammatory response or whether it also participates in disease pathology; thus, determining if $\mathrm{IgE}$ is present in target organs and what role it has in these organs is of interest," explains Rivera. "In addition, whether IgE mediates its effects via basophils or through other cell types, such as plasmacytoid dendritic cells, which are known to express IgE receptors, is unknown." The researchers have initiated a safety trial to investigate whether anti-IgE antibodies might be of therapeutic benefit to patients with SLE.

Susan J. Allison
Original article Dema, B. et al. Immunoglobulin E plays an immunoregulatory role in lupus. J. Exp. Med. doi:10.1084/ jem.20140066 